This is an un-edited version of the original publication published in Physik in unserer Zeit:

https://doi.org/10.1002/piuz.201901534

MARINE HITZEWELLEN | KLIMAFORSCHUNG

((Subhead:)) Hitzewellen im Ozean

\title{
Die unterschätzte Gefahr
}

\section{Thomas Frölicher}

Hitzewellen im Ozean treten heute doppelt so häufig auf wie noch vor 35 Jahren. Und laut Klimamodellvorhersagen sollen marine Hitzewellen in den kommenden Jahrzenten nochmals deutlich zunehmen. Dadurch sind ganze Ökosysteme bedroht.

Der Sommer 2018 präsentierte sich in weiten Teilen der Nordhalbkugel als sehr heiß und trocken. Europa, Nordamerika und Gebiete in Ostasien wurden fast zeitgleich von drei großflächigen Hitzewellen erfasst. Wälder brannten, Flüsse trockneten aus, Felder waren kaum zu bewirtschaften, und viele Menschen mussten wegen der großen Hitze medizinische Hilfe in Anspruch nehmen [1].

2018 wird wohl als eines der wärmsten Jahre und mit neuen Temperaturrekorden in die Geschichte eingehen. Dies ist jedoch nicht erstaunlich, hat doch der Weltklimarat (IPCC, Intergovernmental Panel on Climate Change) bereits 2012 davor gewarnt, dass solche Hitzewellen aufgrund des Klimawandels immer häufiger auftreten werden und dies weitreichende Folgen haben kann [2]. Dass der Klimawandel auch Hitzewellen im Ozean verursachen kann, war damals noch kaum bekannt. Einige außergewöhnliche Hitzewellen im Ozean haben nun aufhorchen lassen. Denn deren Folgen sind nicht nur für die marinen Lebewesen (Abbildung 1), sondern auch für die Menschheit spürbar. Marine Hitzewellen sind Perioden von extrem warmen Meerestemperaturen, die Tage bis Monate anhalten, sich über mehrere tausend Kilometer ausbreiten und mehrere hundert Meter in die Tiefe ausdehnen können [3].

\section{Weltweite Beobachtung}

Marine Hitzewellen wurden über die letzten Jahre in allen Ozeanbecken beobachtet (Abbildung 2). Bereits im Juni 2003 stellte man im Mittelmeer erstmals eine Hitzewelle fest. Damals lagen dessen Oberflächentemperaturen bis zu $3{ }^{\circ} \mathrm{C}$ über dem Mittelwert der früheren Sommertemperaturen. Eine weitere nennenswerte Hitzewelle traf die australische Westküste um die Jahreswende 2010-11. Die Meerestemperaturen übertrafen die Normalwerte für diese Jahreszeit um bis zu $5{ }^{\circ} \mathrm{C}$.

Die wohl bekannteste marine Hitzewelle, eine ungewöhnlich lang andauernde Warmwasserblase mit dem Spitznamen „Blob“, hatte sich vom Winter 2013-14 bis 2016 auf der Wasseroberfläche des Nordostpazifiks ausgebreitet. Blob hatte zeitweise einen Durchmesser von bis zu $1600 \mathrm{~km}$ mit Wassertemperaturen von mehr als $6{ }^{\circ} \mathrm{C}$ über dem langjährigen Durchschnitt. Doch das sind bei Weitem nicht alle bekannten marinen 
Hitzewellen. Die Liste solcher Vorkommnisse kann beliebig erweitert werden. Auch im Golf von Alaska, im Nordwestatlantik, in der Tasmansee, vor der Küste Perus und im Gelben Meer traten Hitzewellen auf. Der tropische Westpazifik und das Korallenmeer wurden sogar mehrmals von Hitzewellen heimgesucht (1998, 2010, 2014-17).

Wie entstehen marine Hitzewellen?

Auf dem Land sind stationäre Wetterlagen und Zusatzeffekte wie ein trockener Boden oft der Auslöser für Hitzeperioden. Im Ozean gibt es eine ganze Reihe von Prozessen, die zu marinen Hitzewellen führen können. Diese Prozesse sind aber bislang weit weniger gut erforscht und quantifiziert als jene über dem Land.

Der wichtigste Treiber von marinen Hitzewellen weltweit gesehen ist El Niño [4], da er großflächig alle paar Jahre die Wassertemperatur erhöht, insbesondere im äquatorialen Pazifik und im Indischen Ozean. El Niño ist das Resultat einer starken Wechselwirkung zwischen der Atmosphäre und dem Ozean und führt durch schwächere Passatwinde zu einem geringeren Auftrieb von kühlem Wasser aus der Tiefe des Ozeans. Das Oberflächenwasser, insbesondere im äquatorialen Pazifik, kann sich so stärker erwärmen.

Einige Hitzewellen haben den Ursprung in atmosphärischen Störungen, zum Beispiel durch ungewöhnlich stabile Wetterlagen oder Änderungen der Winde, die sich über positive Rückkopplungseffekte mit der warmen Meerestemperatur verstärken. Ein sehr stabiles Hochdruckgebiet über dem Nordpazifik führte wohl durch Rückkoppelungseffekte zum erwähnten Blob [5]. Dieses Hochdruckgebiet blockierte den arktischen Winterstürmen den Weg, sodass sie den Nordostpazifik nicht wie gewohnt durchmischen und kühlen konnten.

Das Gleiche gilt für die marine Hitzewelle an der Küste Perus im Jahr 2017, wo sich lokale Südwinde abgeschwächt haben und über Wolkeneffekte eine neue stabile Wetterlage entstand. Eine Verschiebung der Windsysteme über dem Indopazifik verursachte 2011 eine Südwärtsverschiebung des warmen Leeuwinstroms. Dadurch erreichte warmes Wasser weit südlichere Teile der Westküste Australiens als normal und führte dort zu einer weiteren marinen Hitzewelle.

Auch Hitzewellen über dem Land können die Meerestemperaturen schlagartig erhöhen. Die europäische Hitzewelle im 2003 zum Beispiel sorgte für eine erhöhte Wärmeaufnahme des Mittelmeeres, was zu der schon erwähnten marinen Hitzewelle im Juni 2003 führte. Natürlich kann es auch zu rein ozeangetriebenen Hitzewellen kommen, die zum Beispiel durch turbulente Strömungen - dem sogenannten ozeanischen Wetter - im Meer verursacht werden.

\section{Die Erwärmung des Ozeans}

Durch den Anstieg der Treibhausgas-Konzentration in der Atmosphäre hat sich die gespeicherte Wärme im Erdsystem markant erhöht. Der größte Teil der zusätzlichen Wärme wurde vom Ozean aufgenommen und hat signifikant zum Meeresspiegelanstieg beigetragen (siehe „Hochaufgelöste Temperaturmessungen im Ozean“ auf S. und Physik in unserer Zeit 2013, 44(6), 280). Zwischen 1970 und 2010 hat der Ozean $93 \%$ oder 274 ZJ (1 Zettajoule $=10^{21}$ Joule) der zusätzlichen Wärme gespeichert [6]. Nur $7 \%$ der Wärme verteilen sich auf die Atmosphäre, das Land und ist an der Eisschmelze beteiligt.

Durch seine hohe Wärmekapazität und sein großes Volumen ist der Ozean der größte Wärmespeicher im Klimasystem. Er spielt daher eine Schlüsselrolle in der Regulierung des Klimasystems und verlangsamt deutlich die Erwärmungsrate der Atmosphäre. Die hohe 
Wärmeaufnahme des Ozeans hat aber dazu geführt, dass sich seine obersten Schichten kontinuierlich erwärmt haben. Heute liegt daher die Oberflächentemperatur um etwa $0,35^{\circ} \mathrm{C}$ über dem Mittelwert der letzten 35 Jahre (Abbildung 3a). 2017 war das wärmste Jahr seit Beginn der Satellitenmessungen im Jahr 1981. Doch auch die Tiefsee unterhalb von $2000 \mathrm{~m}$ Tiefe zeigt einen deutlichen Trend hin zu wärmeren Temperaturen.

\section{Zunahme von marinen Hitzewellen}

Der schleichende Temperaturanstieg des Ozeans macht auch häufigere und stärkere marine Hitzewellen wahrscheinlicher. Mit Hilfe von Satellitenmessungen der MeeresoberflächenTemperatur konnte eine wissenschaftliche Kooperation in der Schweiz, die meine Gruppe von der Klima- und Umweltphysik der Universität Bern initiiert und geführt hat, zeigen, dass marine Hitzewellen immer häufiger werden [4, 7]. Die Wahrscheinlichkeit mariner Hitzetage hat sich zwischen 1982 und 2016 verdoppelt (schwarze Linie in Abbildung 3b). Quantitativ heißt das: 1982 traten marine Hitzewellen noch zweieinhalb Mal pro Jahr auf, heute sind es fünf Mal [7].

Hitzewellen werden nicht nur wahrscheinlicher, sie werden auch intensiver, dauern länger an und erstrecken sich über größere Gebiete. Aufgrund der hohen Meerestemperaturen in den Jahren 2015 und 2016 erlebte ein Viertel der globalen Meeresoberfläche entweder die intensivste oder längste je gemessene Hitzewelle [3]. Doch nicht nur der offene Ozean ist betroffen, auch in Küstengebieten hat die Anzahl Hitzetage markant zugenommen [8]. Aber wie kann man die Zunahme von marinen Hitzewellen über die jüngsten Jahrzehnte erklären?

Mit Hilfe von Klimamodellen haben wir untersucht, ob natürliche Klimaschwankungen wie die El-Niño-Südoszillation und die Atlantische Meereszirkulation für die Änderungen in der Anzahl Hitzetage verantwortlich sind - oder aber anthropogene Antriebe wie die Zunahme der Treibhausgas-Konzentration in der Atmosphäre. Dazu haben wir KlimamodellSimulationen sowohl mit („,Klimamodelle mit anthropogenen Teibhausgasen“ in Abbildung 4) als auch ohne menschengemachte Treibhausgas-Konzentrationen (,Klimamodelle ohne anthropogene Treibhausgase" in der Abbildung) berechnet. Anschließend haben wir diese simulierten Trends in der Wahrscheinlichkeit von Hitzetagen mit den tatsächlichen beobachteten Trends (,Satellitendaten“ in Abbildung 4) verglichen.

Die Ergebnisse zeigen, dass der Großteil der beobachteten Änderungen über die letzten Jahrzehnte nur durch die Simulationen mit menschengemachten TreibhausgasKonzentrationen reproduziert werden können. Bereits heute werden $87 \%$ aller Hitzewellen durch die anthropogen bedingte globale Erwärmung verursacht [7]. Zwar hätten theoretisch einige Hitzewellen auch in der Vergangenheit entstehen können, jedoch wäre die Wahrscheinlichkeit eines Auftretens viel geringer gewesen. Die Hitzewelle im Korallenmeer und im Golf von Alaska im Jahr 2016 ist erstaunlicherweise nicht mehr mit Simulationen ohne menschengemachte globale Erwärmung reproduzierbar [9, 10]. Das heißt, dass diese Hitzewellen mit großer Wahrscheinlichkeit ohne Klimaerwärmung nicht aufgetreten wären.

\section{Zukünftige Entwicklung}

Wegen der langsamen Umstellung der Wirtschaft, speziell der Energiewirtschaft, hin zu einer Senkung des Verbrauchs von Kohlenstoff, sowie der Trägheit des Klimasystems müssen wir befürchten, dass sich das Klima in den nächsten Jahrzehnten weiter erwärmen wird. Dadurch werden auch marine Hitzewellen häufiger vorkommen (Abbildung 5a). 
Neueste Klimamodell-Simulation zeigen, dass die Anzahl der ozeanischen Hitzetage um das 16-Fache zunehmen wird, sofern sich die Erde nicht mehr als $1,5^{\circ} \mathrm{C}$ erwärmt, wie es im Pariser Klimaabkommen als Ziel vereinbart ist [7]. Sollte jedoch eine Erwärmung von $2{ }^{\circ} \mathrm{C}$ eintreten, wird sich die Anzahl Hitzetage um das 23-Fache erhöhen, bei 3,5 ${ }^{\circ} \mathrm{C}$ sogar um das 41-fache. In anderen Worten heißt dies, dass solche Hitzeereignisse vor der Industrialisierung im Schnitt einmal in 100 Tagen vorkamen, bei $1,5^{\circ} \mathrm{C}$ jeden sechsten Tag, bei $2{ }^{\circ} \mathrm{C}$ jeden vierten Tag und bei $3,5^{\circ} \mathrm{C}$ Erwärmung jeden zweiten Tag.

Aber nicht nur die Anzahl der Hitzetage würde in Zukunft zunehmen. Bei 3,5 ${ }^{\circ} \mathrm{C}$ Erwärmung würden die Hitzewellen im Mittel 112 Tage anhalten, und die betroffenen Gebiete würden auf $94,5 \times 10^{5} \mathrm{~km}^{2}$ anwachsen - das entspricht der Fläche von China. Zum Vergleich: Vor der Industrialisierung dauert eine Hitzewelle im Schnitt 11 Tage und erstreckte sich im Mittel über eine Fläche von $4,2 \times 10^{5} \mathrm{~km}^{2}$, was der Schweiz entspricht.

Grundsätzlich sind alle Ozeanregionen von der Zunahme der Hitzewellen betroffen, und dies wird auch in der Zukunft so sein (Abbildung 5b). Die regionalen Unterschiede sind vor allem auf die unterschiedlich starke Erwärmung der verschiedenen Ozeanregionen zurückzuführen. Die deutlichste Zunahme prognostizieren die Klimamodelle in der Arktis und im Westen des tropischen Pazifiks.

In der Arktis werden die Anzahl Hitzetage um das 50-Fache zunehmen, im Westpazifik sogar um das 70-Fache. Die Arktis ist speziell, da dort das Verschwinden des Meereises zu einer verhältnismäßig starken Zunahme der Hitzetage führt. Im tropischen Westpazifik sind die saisonalen und jährlichen Schwankungen in der Meerestemperatur sehr gering. Deswegen führt dort bereits eine kleine Zunahme der Temperatur zu einer großen Änderung in der Anzahl der Hitzetage. Im südlichen Polarmeer hingegen ist nur mit einer kleinen Zunahme an Hitzetagen zu rechnen. Da im Südpolarmeer das Meerwasser aus großer Tiefe aufsteigt, kann sich die Oberfläche nicht so schnell erwärmen, wie das zum Beispiel in den mittleren Breiten passiert.

Interessanterweise nimmt die Anzahl der Hitzewellen im Meer rascher zu als über dem Land [11]. Weil sich Wasser langsamer erwärmt und abkühlt als Luft, schwankt die Temperatur der Ozeane weniger stark als die der Atmosphäre. Dies hat zur Folge, dass die Weltmeere schon bei einer kleineren Temperaturerhöhung eine ungleich größere Änderung an Extremereignissen erfahren. Eine leichte Temperaturzunahme im Meer kommt also dort schon einer Hitzewelle gleich. Aber wie vertrauenswürdig sind diese Prognosen aus Klimamodellen?

Der Vergleich zwischen Satellitenmessungen und Modellvorhersagen von marinen Hitzewellen zeigt, dass die Modelle die beobachteten Änderungen in der Anzahl der Hitzewellen über die letzten 35 Jahre sehr gut abbilden können (rote Linien in Abbildung 3b). Abweichungen zwischen Modellen und Beobachtungen gibt es aber bei der Dauer und der Ausdehnung der Hitzewellen. Dies könnte daran liegen, dass die Auflösung der Modelle noch immer relativ grob ist. Im Schnitt haben die numerischen Ozeanmodelle eine horizontale Gitterweite von hundert Kilometern und können deshalb kleinskalige turbulente Wirbel und Strukturen des Ozeans nicht simulieren. Eine höhere Auflösung der horizontalen Gitterweite wäre dazu nötig. Dies erfordert jedoch enorme Computerressourcen, die heute noch kaum zur Verfügung stehen. 


\section{Marine Ökosysteme bedroht}

Hitzewellen im Ozean können ganze Ökosysteme aus dem Gleichgewicht bringen und stellen damit auch eine Bedrohung für die Fischerei dar. Die Korallen im australischen Great Barrier Riff zum Beispiel sind ein Ökosystem, das sehr empfindlich auf erhöhte Wassertemperaturen reagiert. Unter Hitzestress können die Korallen ausbleichen, was bei einer zu langen Hitzeperiode zum Absterben führen kann.

Korallenbleichen wurden temporär und örtlich begrenzt bereits seit einigen Jahrzehnten beobachtet. 2015 und 2016 kam es wegen der weltweit hohen Meerestemperaturen zu der stärksten je gemessenen globalen Korallenbleiche [12]. Mehr als $70 \%$ der weltweiten Riffe wurden gebleicht und bis zu einem Drittel massiv beschädigt. Zwar haben sich die Korallenriffe bisher oft erholen können. Werden die Abstände zwischen den Hitzewellen jedoch kürzer, haben die Korallen keine Zeit zur Regeneration mehr, und es ist mit irreversiblen Schäden zu rechnen.

Beobachtungen während den marinen Hitzewellen an der Westküste Australiens [13] und im Nordostpazifik [14] haben gezeigt, dass diese nicht nur zum Absterben von Korallen führen, sondern auch andere dramatische biologische Auswirkungen haben. Diese reichen von Veränderungen der Artenzusammensetzung über die geographische Verschiebung von Arten bis zu schädlichen Algenblüten, gestrandeten Säugetieren und im Extremfall zu Massensterben bestimmter Arten.

Die Westküste Australiens ist bekannt für riesige Ansammlungen von Braunalgen. Diese marinen „Algenwälder“ haben ähnliche Funktionen wie terrestrische Regenwälder: Sie bieten vielen Fischen Lebensraum und Nahrungsgrundlage. Die Hitzewelle an Australiens Westküste führte in kürzester Zeit zum Absterben dieser Algenwälder und zu einem neuen Ökosystem mit tropischen Fischen und Seegräsern. Die Algenwälder haben sich bis heute nicht mehr erholt. Der Blob im Nordostpazifik führte unter anderem dazu, dass der Bestand von Grünalgen und Planktonkrebsen massiv zurückging. Etliche Fischarten wanderten in kühlere Regionen ab. Als Folge konnten viele Seevögel keine Nahrung mehr finden und verhungerten. Von der stark erhöhten Wassertemperatur profitierten aber auch einige Organismen, wie zum Beispiel Gift produzierende Kieselalgen.

Auch für die Fischerei hatten vergangene marine Hitzewellen gravierende Folgen. So hat die Hitzewelle im Nordwestatlantik im 2012 dafür gesorgt, dass die Hummersaison Monate früher als sonst begann, als die Fischer und Konsumenten noch gar nicht bereit waren, die Hummer zu fangen und abzunehmen. Die Fischindustrie verbuchte einen großen wirtschaftlichen Verlust - die Preise für Hummer fielen um 70 \% [15]. Die Warmwasserblase im Nordostpazifik führte dazu, dass Teile der kommerziellen und Freizeit-Fischerei geschlossen wurden, was auch zu einem Verlust von mehreren Millionen Dollar führte [14].

Auch die Tourismusbranche kann durch marine Hitzewellen Einbußen verzeichnen. Die Ausbreitung von giftigen Kieselalgen kann zu Schließungen von ganzen Küstenabschnitten führen und auch für den Menschen gesundheitsschädigende Auswirkungen haben. Sollte zudem das Korallensterben in Australien weiter anhalten und dadurch die Vielfalt der Meeresbewohner massiv abnehmen, könnte die Anzahl Touristen beim Great Barrier Reef massiv einbrechen.

\section{Ausblick}

Während der globale Temperaturanstieg des Ozeans und der damit verbundene Anstieg des Wasserspiegels seit Jahren erforscht wird, ist über Extreme wie marine Hitzewellen und deren 
Auswirkungen bisher nur wenig bekannt [11]. Die hier erwähnten Beispiele zeigen jedoch mit klarer Deutlichkeit, dass eine ganze Kette von Organismen und Ökosystemen durch marine Hitzewellen betroffen sind. Da mit einer weiteren Zunahme von Hitzewellen zu rechnen ist, werden auch Ökosysteme immer größerem Stress ausgesetzt. Insbesondere Meereslebewesen, die nicht mobil sind und sich nicht anpassen können, werden daher stark betroffen sein.

Beobachtungen und Modellsimulationen zeigen zudem, dass auch andere Faktoren wie Ozeanversauerung und Sauerstoffverlust die marinen Lebewesen und Ökosysteme aus dem Gleichgewicht bringen können [16]. Über die Auswirkungen der verschiedenen Stressoren auf einzelne Arten oder ganze Ökosysteme und deren sozioökonomischen Bedeutungen wissen wir aber noch wenig. Dazu braucht es weiterführende interdisziplinäre Forschung.

\section{Zusammenfassung}

Marine Hitzewellen - längere Perioden mit extrem hohen Meerestemperaturen - haben in den vergangenen Jahrzehnten stark zugenommen. Die Anzahl der marinen Hitzetage hat sich in den letzten 35 Jahren verdoppelt. Hitzewellen wurden auch intensiver, länger und erstrecken sich über größere Gebiete. Diese Trends werden sich infolge des Klimawandels weiter verstärken. Die Folgen für die Ökosysteme der Weltmeere sind gravierend. Besonders bedroht sind sesshafte Organismen, die sich den wärmeren Bedingungen nicht anpassen können.

\section{Stichwörter}

Marine Hitzewelle, Klimaerwärmung, Treibhausgas-Konzentration, Representative Concentration Pathway, Klimamodell, Simulation, marines Ökosystem, Argo-Boje.

\section{Danksagung}

Der Autor bedankt sich bei Charlotte Laufkötter für Abbildung 1 und beim Schweizer Nationalfonds für die finanzielle Unterstützung (PP00P2_170687).

\section{Literatur}

[1] WMO, July sees extreme weather with high impacts, 1 August 2018, http://t1p.de/5yhy

[2] S. I. Seneviratne et al., in: Managing the Risks of Extreme Events and Disasters to Advance Climate Change Adaptation, United Nations, New York 2012, S. 109, online: http://tlp.de/ihim

[3] A. J. Hobday et al., Prog. Oceanogr. 2016, 141, 227.

[4] E. C. J. Oliver et al., Nat. Commun. 2018, 9, 1324.

[5] E. Di Lorenzo, N. Mantua, Nat. Clim. Chang. 2016, 6, 1.

[6] L. Cheng et al., Sci. Adv. 2017, 3, 1.

[7] T. L. Frölicher, E. M. Fischer, N. Gruber, Nature. 2018, 560, 360.

[8] F. P. Lima, D. S. Wethey, Nat. Commun. 2012, 3, 1.

[9] J. E. Walsh et al., Bull. Am. Meteorol. Soc. 2018, 98, 39.

[10] E. C. J. Oliver et al., Bull. Am. Meteorol. Soc. 2018, 99, S44.

[11] T. L. Frölicher, C. Laufkötter, Nat. Commun. 2018, 9, 650.

[12] T. P. Hughes et al., Nature. 2017, 543, 373.

[13] T. Wernberg et al., Science. 2016, 353, 169.

[14] L. Cavole et al., Oceanography. 2016, 29, 273.

[15] K. E. Mills et al., Oceanography. 2014, 26, 191.

[16] T. L. Frölicher et al., Global Biogeochem. Cycles. 2016, 30, 1224. 


\section{Der Autor}

Thomas Frölicher ist Förderprofessor des Schweizerischen Nationalfonds in der Abteilung für Klima- und Umweltphysik der Universität Bern. Dort leitet er die Gruppe

Ozeanmodellierung. Nach seiner Dissertation an der Universität Bern forschte er an der Princeton University und der ETH Zürich. Er ist Hauptautor im Spezialbericht zu „Ozean und Kryosphäre" des Weltklimarats IPCC.

\section{Anschrift}

Prof. Dr. Thomas Frölicher, Universität Bern, Klima- und Umweltphysik und Oeschger Zentrum, Sidlerstrasse 5, 3012 Bern, Schweiz.

\section{((Bildunterschriften))}

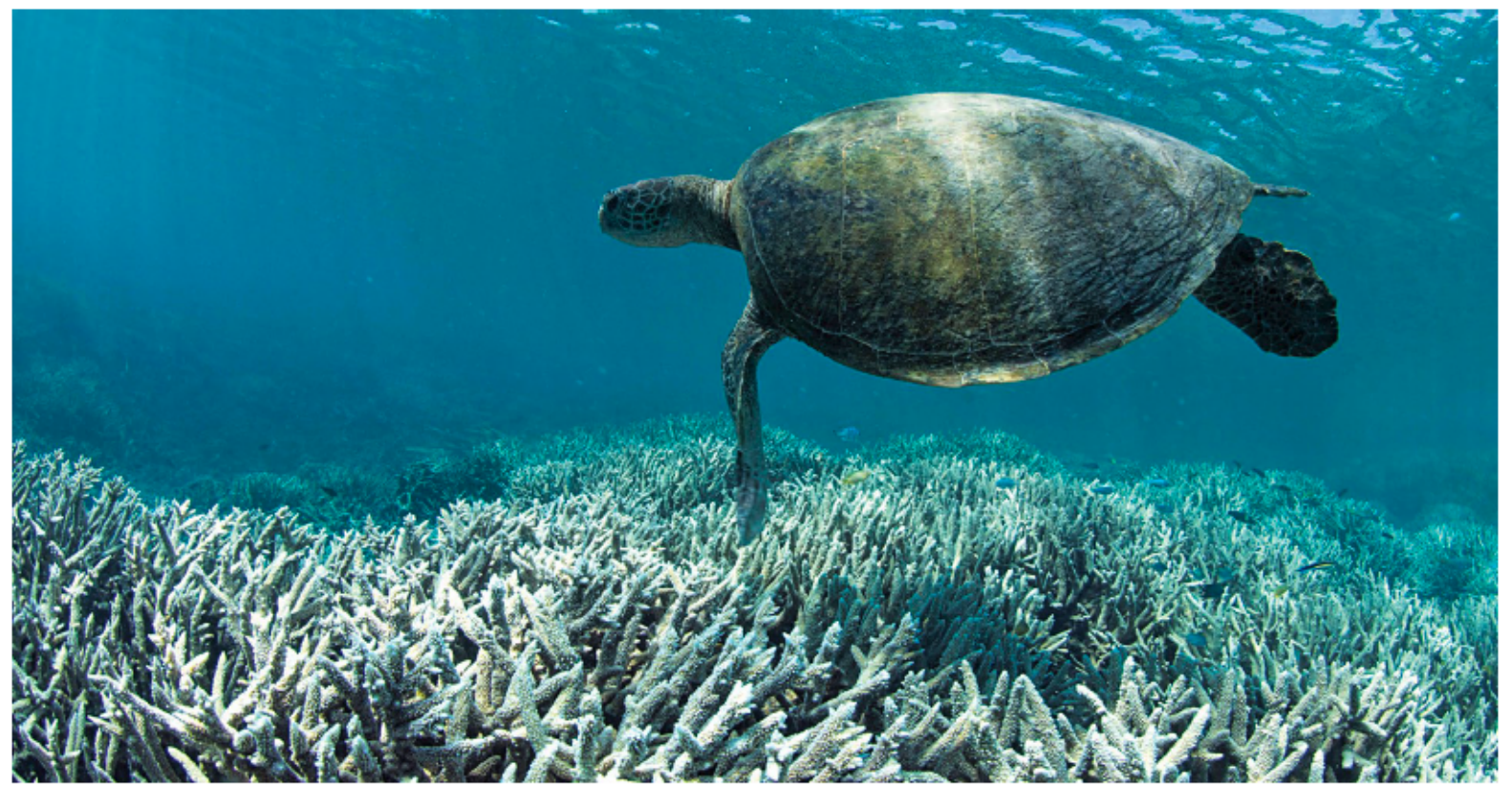

Abb. 1 Korallen im südlichen Teil des Great Barrier Riffs nach der globalen

Korallenbleiche im Februar 2016 (Foto: The Ocean Agency / XL Catlin Seaview Survey). 


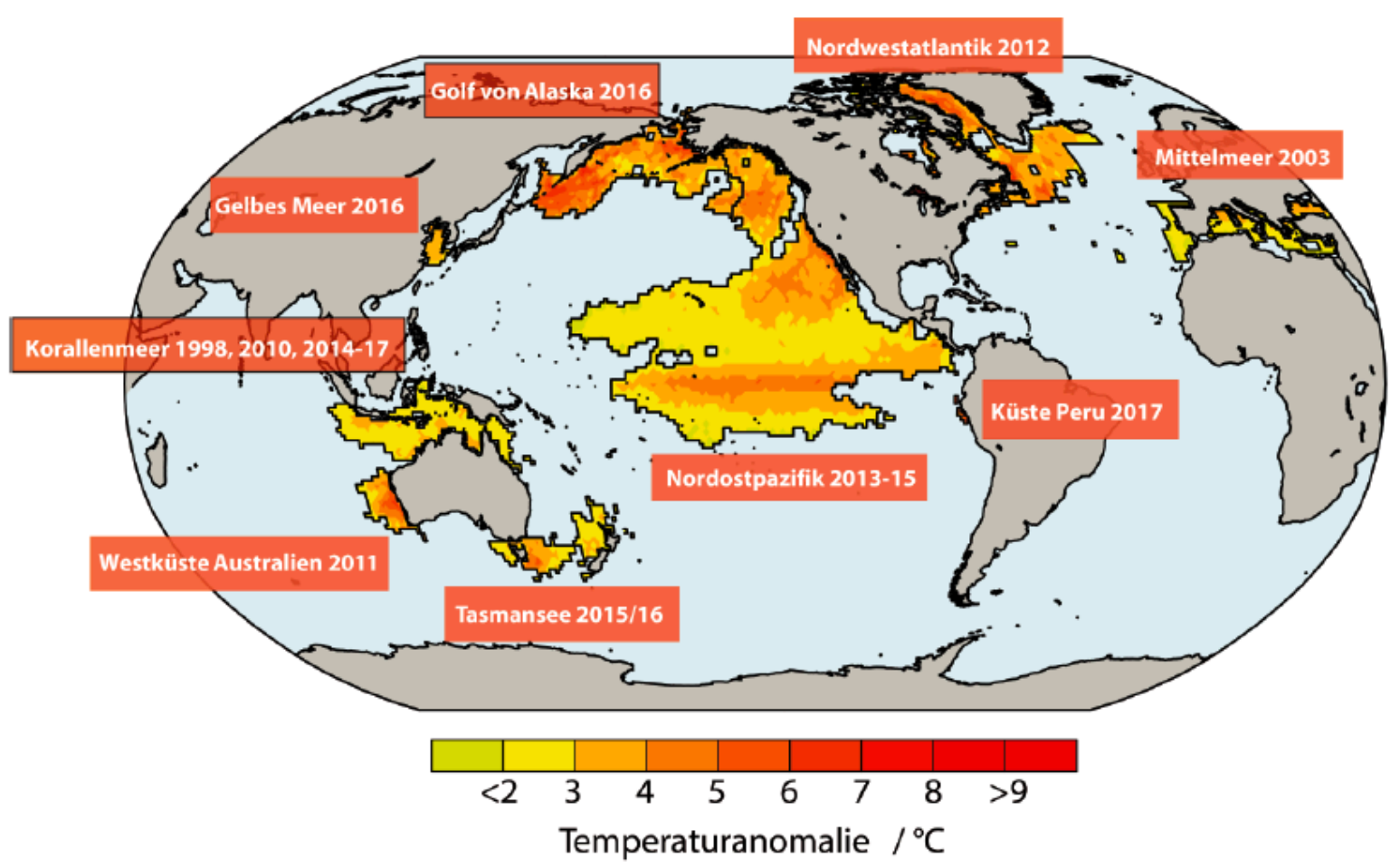

ABB. 2 MARINE HITZEWELLEN

Räumliche Ausdehnung und Intensität der beobachteten marinen Hitzewellen über die letzten 15 Jahre (modifiziert nach [11]). Gezeigt sind die beobachteten maximalen

Temperaturanomalien während den Hitzewellen. Die Kästchen beschreiben den Ort und das Auftrittsjahr der Hitzewelle. 




\section{ABB. 3 MEERESOBERFLÄCHEN-TEMPERATUR}

Änderung der globalen Meeresoberflächen-Temperatur und der Wahrscheinlichkeit mariner Hitzewellen seit 1982 (modifiziert nach [7]). a) Die Anomalie der beobachteten

Meeresoberflächen-Temperatur relativ zum Mittel der Periode 1982-2016 $(\Delta \mathrm{T}(\mathrm{t})=\mathrm{T}(\mathrm{t})$ $\mathrm{T}_{1982-2016) .}$ b) Die Wahrscheinlichkeit einer Hitzewelle gegenüber dem Referenzzustand 19822016. Die schwarzen Linien zeigen Satellitendaten, die roten Resultate von zwölf verschiedenen Klimamodellen. Die dicke gestrichelte rote Linie zeigt das Mittel dieser Modellsimulationen. 


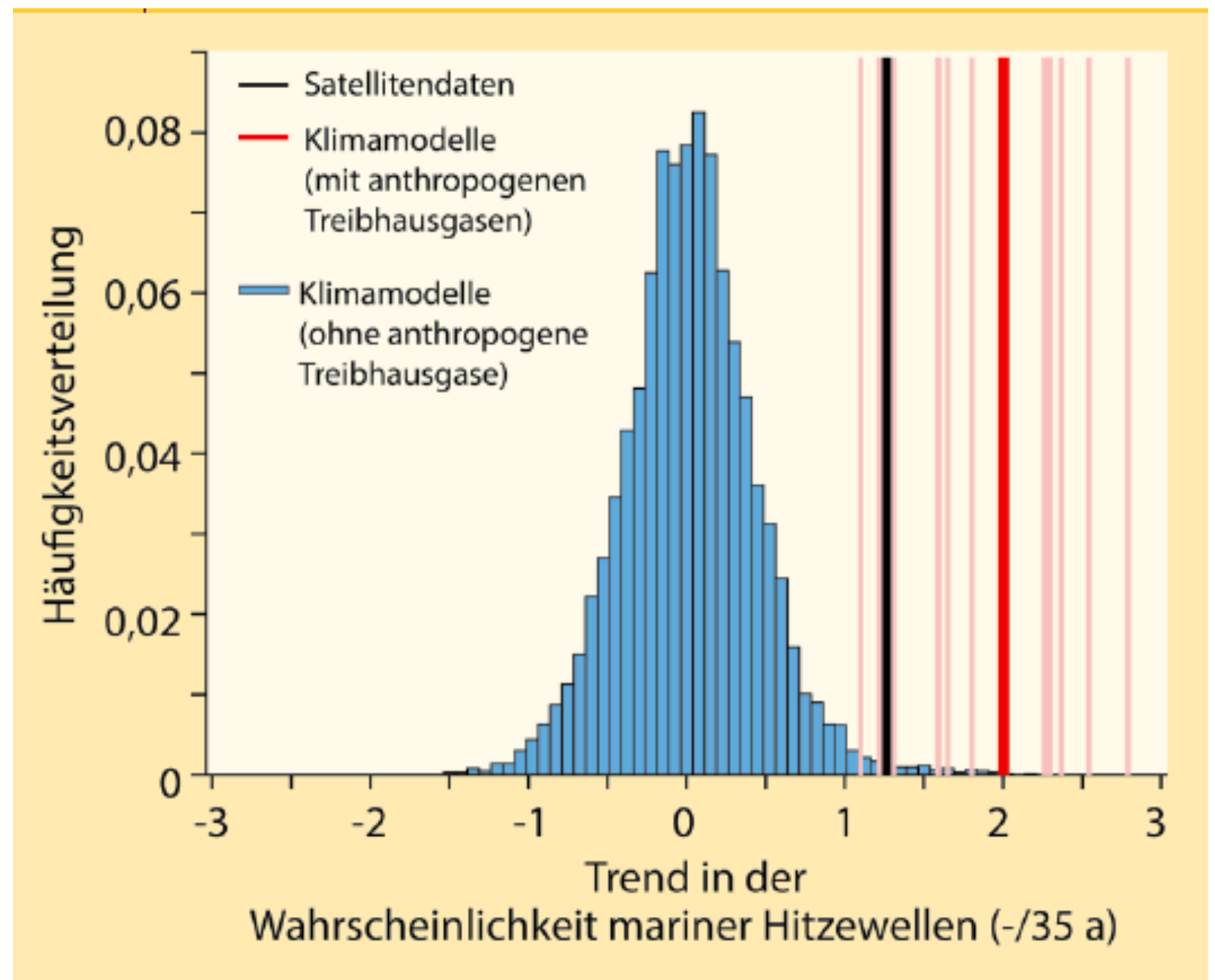

ABB. 4 TRENDS MARINER HITZWELLEN

Beobachtete und modellierte Trends in marinen Hitzewellen über die Satellitenperiode (aus [7]). Die Häufigkeitsverteilung zeigt zum Vergleich die Trends von marinen Hitzewellen über 35 Jahre, die alle zwölf Klimamodelle ohne anthropogene Treibhausgase simuliert haben.

Den Trend in der Wahrscheinlichkeit berechnen wir entsprechend der Satellitenperiode nach $\mathrm{P}=\mathrm{P} 1(\mathrm{t}) / \mathrm{P}(1982-2016)$, so kommen die Zahlenwerte an der unteren Achse zustande. Ein Trend von 2 bedeutet also, das sich die Anzahl der Hitzewellen über 35 Jahre verdoppelt hat. Die schwarze Linie steht für den von Satelliten beobachteten Trend zwischen 1982 und 2016. Die roten Linien zeigen die simulierten Trends in marinen Hitzewellen zwischen 1982 und 2016 der zwölf Klimamodell-Simulationen, die mit anthropogenen TreibhausgasKonzentrationen gerechnet wurden.

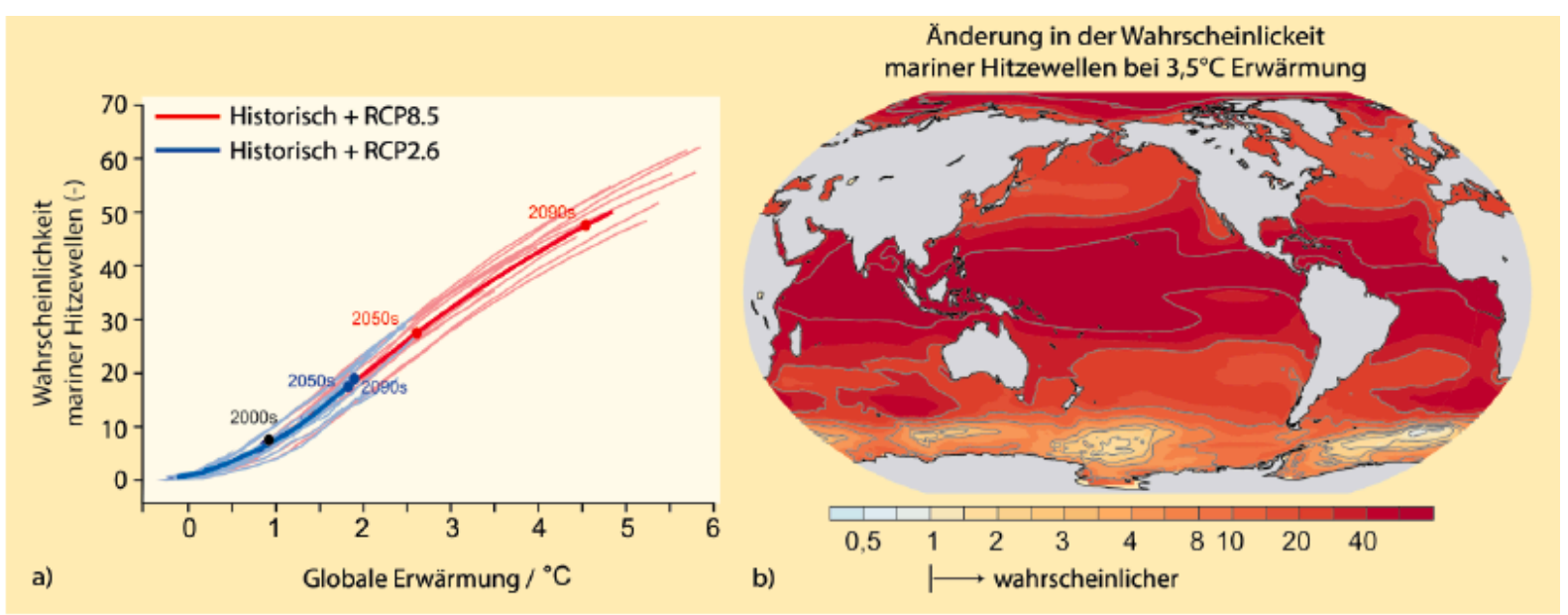




\section{ABB. 5 SZENARIEN}

Globale und regionale Änderung der Wahrscheinlichkeit von marinen Hitzewellen unter verschiedenen globalen Temperaturänderungen seit der vorindustriellen Zeit (nach [7]). a) Die dünnen blauen und roten Linien zeigen Resultate von zwölf verschiedenen Klimamodellen für die Periode 1861 - 2100. Die dicke rote und blaue Linie zeigen das Mittel dieser Simulationen für die Szenarien RCP2.6 und RCP8.5. RCP: Representative Concentration Pathways; diese Repräsentativen Konzentrationspfade beschreiben verschiedene Szenarien für die Entwicklung der Treibhausgas-Konzentrationen, RCP2.6 steht für einen sehr geringen, RCP8.5 einen hohen Zuwachs. b) Die simulierte Änderung der Wahrscheinlichkeit mariner Hitzewellen unter 3,5 $\mathrm{C}$ globaler Erwärmung.

\section{((Informationskasten:))}

\section{Hochaufgelöste Temperaturmessungen im Ozean}

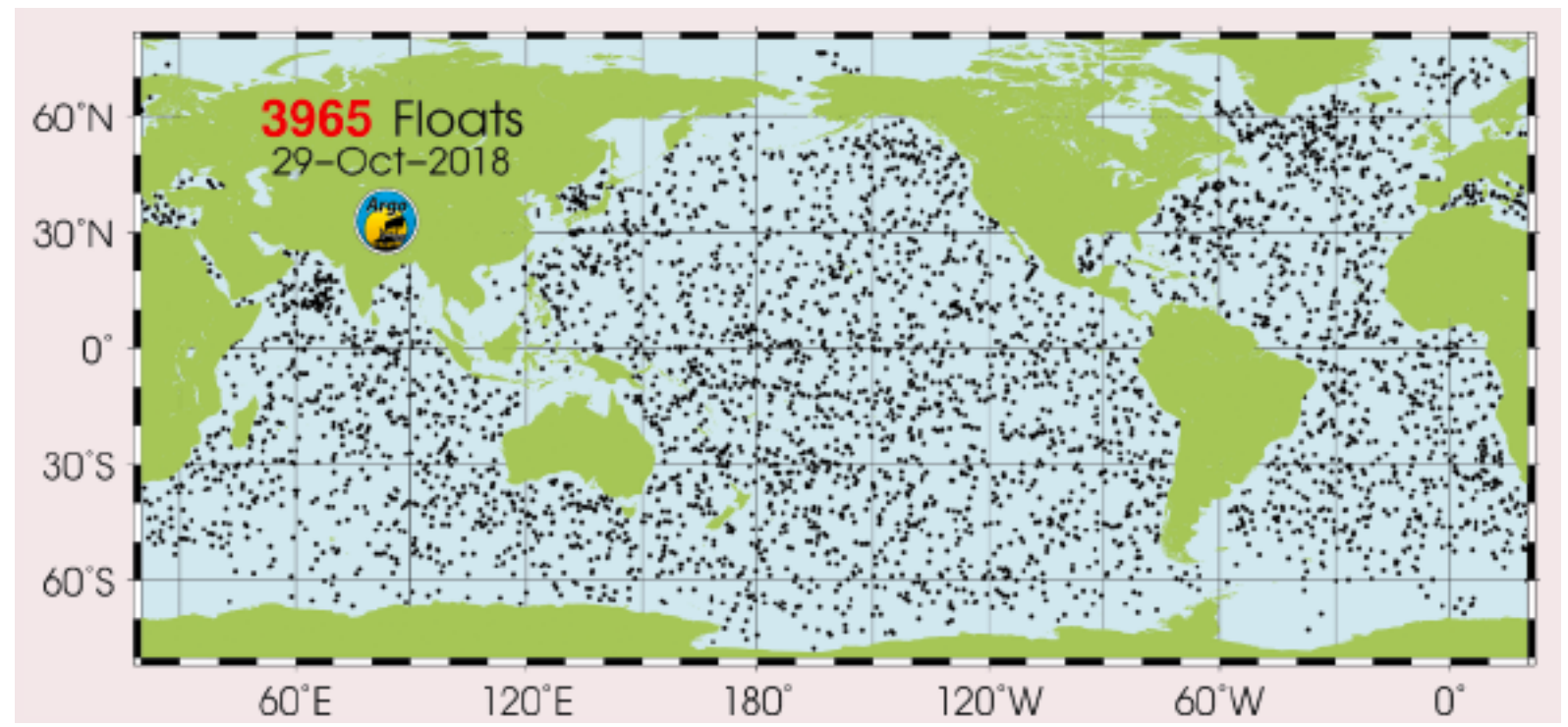

A Weltweite Verteilung der 3965 Bojen des Argo-Messnetzes am 29. Oktober 2018. 


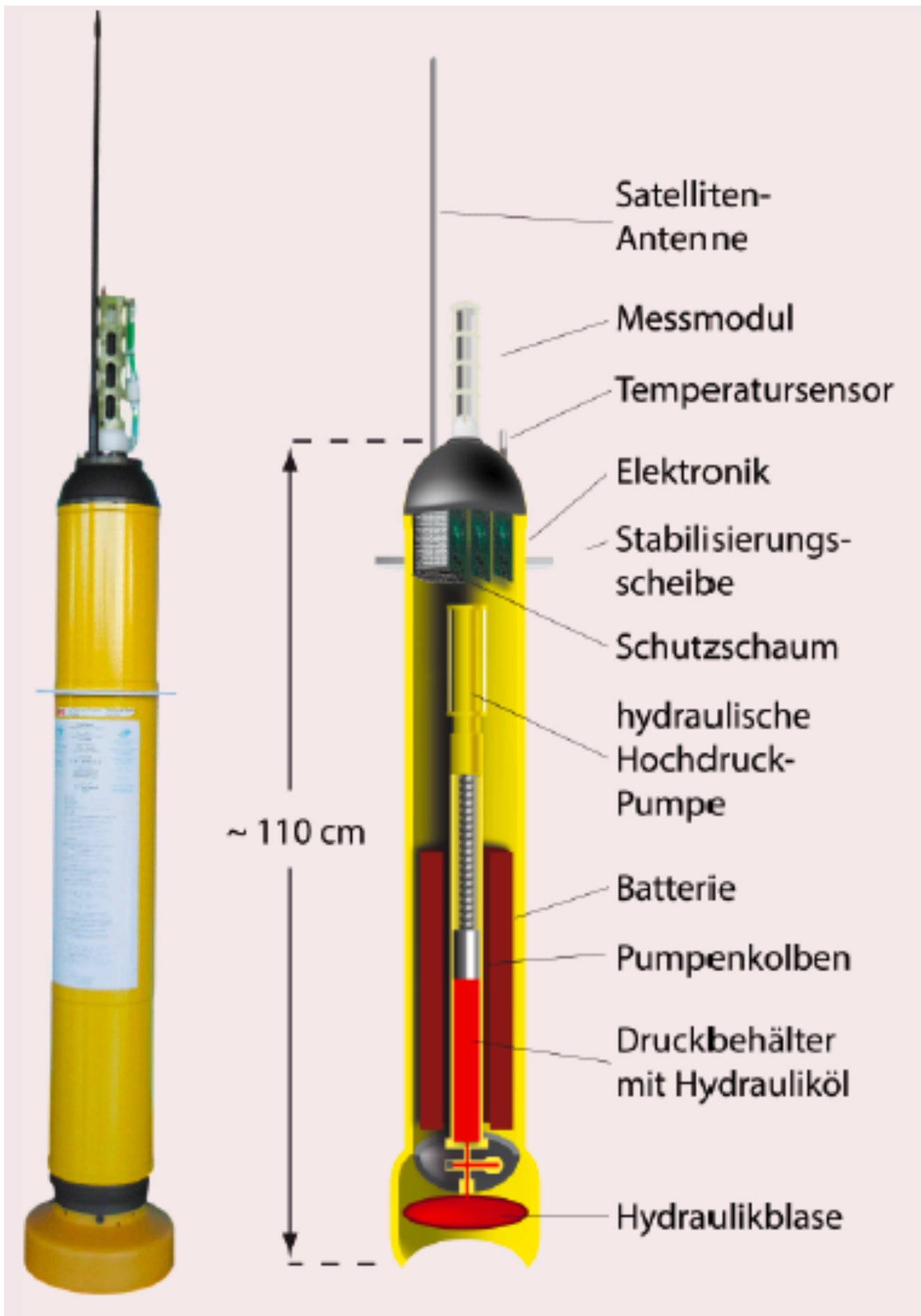

B Eine typische Argo-Messboje ist etwas mehr als 1 m hoch, hat einen Durchmesser von circa $14 \mathrm{~cm}$ und ist etwa $25 \mathrm{~kg}$ schwer (Grafik: R. Wengenmayr).

Konventionell erfolgen Temperaturmessungen im Ozean vorwiegend durch ortsfest installierte Bojen oder auf Schiffen. Um jedoch relativ kleinräumige und kurzlebige Hitzewellen im Ozean zu untersuchen, braucht es Messsysteme, die räumlich und zeitlich hochaufgelöste Daten liefern können. Erst die Satellitentechnik und eine Flotte von sogenannten Argo-Treibbojen ermöglichte die Erforschung von Hitzewellen im Ozean.

Die Meeresoberflächen-Temperatur wird seit 1981 mit Satelliten mit Radiometern im Infrarot- oder Mikrowellenbereich gemessen. Messungen aus dem Inneren des Ozeans konnten jedoch erst die Argo-Bojen liefern. Fast 4000 solcher Messbojen treiben seit der 
Jahrtausendwende in allen Ozeanregionen, um die Temperatur und andere Größen wie Salzgehalt und Druck zu messen (Abbildung A). Das Argo-Netzwerk wird von mehr als 30 Nationen aus aller Welt betrieben und zum Großteil durch die USA finanziert.

Argo-Bojen sind autonome Tauchroboter (Abbildung B). Die meiste Zeit treiben sie in einer Parktiefe von etwa $1000 \mathrm{~m}$. Alle zehn Tage taucht eine solche Boje zunächst auf $2000 \mathrm{~m} \mathrm{ab}$, um anschließend von dort an die Meeresoberfläche aufzusteigen. Unterwegs misst sie Temperatur, Salzgehalt und Druck, denn aus diesen Daten lässt sich unter anderem die Dichte des Meerwassers ermitteln - und horizontale und vertikale Dichteunterschiede treiben großskalige Meeresströmungen an. Die aufgezeichneten Daten funkt sie an Zentralen in den USA, Australien, Japan und Frankreich, wo diese geprüft und zur Nutzung nahezu in Echtzeit bereitgestellt werden. Anschließend beginnt die Prozedur von vorne. Die Boje sinkt auf Parktiefe ab, um dort erneut zehn Tage mit der Strömung zu gleiten. Aus ihren Funkpositionen lässt sich so das mittlere Strömungsfeld in Parktiefe ermitteln.

Um wie ein kleines U-Boot operieren zu können, nutzt eine Argo-Boje Öl. Das Öl kann sie mit einer Hochdruckpumpe zwischen einer Kunststoffblase und einem Druckbehälter hin und her pumpen. Wenn sie von der Parktiefe in $2000 \mathrm{~m}$ Tiefe absteigen will, pumpt sie etwas Ö1 aus der Blase in den Druckzylinder, zum Aufstieg macht sie es umgekehrt. Dabei genügen schon kleine Volumenveränderungen der Blase. Zum Aufstieg aus $2000 \mathrm{~m}$ benötigt die Boje weniger als $1 \%$ Volumenvergrößerung und eine entsprechend geringe Dichteverringerung, denn die Dichte des Meerwassers ist in dieser Tiefe im Durchschnitt nur $3 \mathrm{~kg} / \mathrm{m}^{3}$ höher ist als an der Oberfläche: 1027 anstatt $1024 \mathrm{~kg} \mathrm{~m}^{-3}$.

Die Lebensdauer einer Argo-Boje beträgt vier bis sechs Jahre, bis die Batterien leer sind. Die meisten Bojen sinken dann auf den Ozeanboden, da die Außenhülle mit der Zeit undicht wird; ein kleiner Teil gerät an Land, einige Bojen davon werden an die angegebene Adresse geschickt. Die neueste Generation ist mit Extrasensoren ausgerüstet, die Sauerstoff, Chlorophyll, Nährstoffe und pH messen können. Einige Spezialbojen erreichen sogar Tauchtiefen unterhalb $2000 \mathrm{~m}$ und können so Änderungen im tiefen Ozean untersuchen. 